\title{
Peri-ictal network dynamics of spike-wave discharges: Phase and spectral characteristics
}

\author{
Annika Lüttjohann ${ }^{\mathrm{a}, *}$, Jan-Mathijs Schoffelen ${ }^{\mathrm{b}, \mathrm{c}}$, Gilles van Luijtelaar ${ }^{\mathrm{a}}$ \\ a Donders Institute for Brain, Cognition and Behaviour, Donders Centre for Cognition, Radboud University Nijmegen, Nijmegen, The Netherlands \\ ${ }^{\mathrm{b}}$ Donders Institute for Brain, Cognition and Behaviour, Donders Centre for Cognitive Neuroimaging, Radboud University Nijmegen, Nijmegen, The Netherlands \\ c Max Planck Institute for Psycholinguistics, Nijmegen, The Netherlands
}

\section{A R T I C L E I N F O}

\section{Article history:}

Received 14 August 2012

Revised 19 October 2012

Accepted 24 October 2012

Available online 1 November 2012

\section{Keywords:}

Cortico-thalamo-cortical system

Network interactions

Pairwise-phase-consistency

Time-frequency-analysis

Epileptic precursor activity

Reticular-thalamic-nucleus

Posterior thalamus

Somatosensory cortex

Spike-wave discharges

WAG/Rij rats

\begin{abstract}
A B S T R A C T
Purpose: The brain is a highly interconnected neuronal assembly in which network analyses can greatly enlarge our knowledge on seizure generation. The cortico-thalamo-cortical network is the brain-network of interest in absence epilepsy. Here, network synchronization is assessed in a genetic absence model during 5 s long pre-ictal->ictal transition periods.

Method: 16 male WAG/Rij rats were equipped with multiple electrodes targeting layer 4 to 6 of the somatosensory-cortex, rostral and caudal RTN, VPM, anterior-(ATN) and posterior (Po) thalamic nucleus. Local field potentials measured during pre-ictal->ictal transition and during control periods were subjected to time-frequency and pairwise phase consistency analysis.

Results: Pre-ictally, all channels showed spike-wave discharge (SWD) precursor activity (increases in spectral power), which were earliest and most pronounced in the somatosensory cortex. The caudal RTN decoupled from VPM, Po and cortical layer 4. Strong increases in synchrony were found between cortex and thalamus during SWD. Although increases between cortex and VPM were seen in SWD frequencies and its harmonics, boarder spectral increases (6-48 Hz) were seen between cortex and Po. All thalamic nuclei showed increased phase synchronization with Po but not with VPM.

Conclusion: Absence seizures are not sudden and unpredictable phenomena: the somatosensory cortex shows highest and earliest precursor activity. The pre-ictal decoupling of the caudal RTN might be a prerequisite of SWD generation. Po nucleus might be the primary thalamic counterpart to the somatosensory-cortex in the generation of the cortico-thalamic-cortical oscillations referred to as SWD.
\end{abstract}

(C) 2012 Elsevier Inc. All rights reserved.

\section{Introduction}

Childhood absence epilepsy is a neurological disorder found in children between the ages of 5 to 12. Its main clinical symptom is the frequent occurrence of periods of reduced consciousness and unresponsiveness which are accompanied by concomitant, highly synchronous, bilateral spike and wave discharges (SWD), recorded in the electroencephalogram (Depaulis and van Luijtelaar, 2006). For a long time, absence seizures have been categorized as a primary

\footnotetext{
Abbreviations: SWD, spike-wave-discharges; ctx4, layer 4 of the somatosensory cortex; ctx5, layer 5 of the somatosensory cortex; ctx6, layer 6 of the somatosensory cortex; ATN, anterior thalamic nucleus; Po, posterior thalamic nucleus; VPM, ventral-postero-medial nucleus of the thalamus; cRTN, caudal reticular thalamic nucleus; rRTN, rostral reticular thalamic nucleus; FCTS, first cortico-thalamic spike; PPC, pairwise-phase-consistency; TFA, time-frequency analysis.

* Corresponding author at: Donders Centre for Cognition, Radboud University Nijmegen, PO Box 9104, 6500 HE Nijmegen, The Netherlands.

E-mail address: a.luttjohann@donders.ru.nl (A. Lüttjohann).
}

generalized form of seizures (ILAE, 1989) occurring unpredictably and simultaneously in the whole brain. However, with advances in techniques it becomes more and more apparent that all kinds of seizures, including absence seizures, can better be regarded as occurring in (selective) rapidly interacting brain networks, currently termed 'system epilepsy' (Berg et al., 2010; Capovilla et al., 2009).

The study of such seizure networks can greatly enlarge our knowledge on seizure generation. It can help to elucidate the mechanisms of communication between and within brain networks involved in seizure occurrence (how different brain regions communicate (interact) during seizure generation) and might show whether there are crucial brain regions (hubs) and loops that are more important than others.

One frequently suggested way of neuronal communication is through coherent brain oscillations (coherence) (Buzsaki, 2006). Coherence estimates the phase-stability between two signals across trials and might therefore be seen as a measure of neural synchrony (Vinck et al., 2010).

For absence epilepsy, strong cortico-thalamic coherence and neuronal firing phase locked to the rhythmic spike and wave cycle 


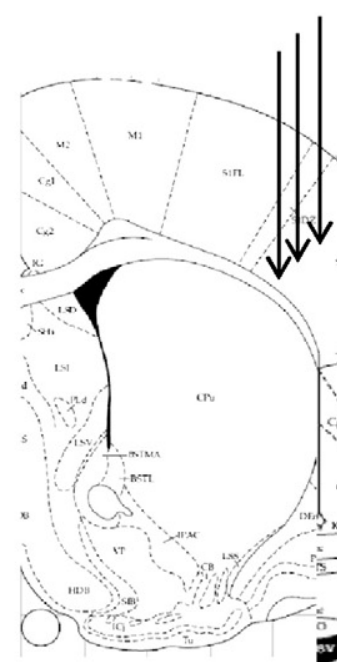

A

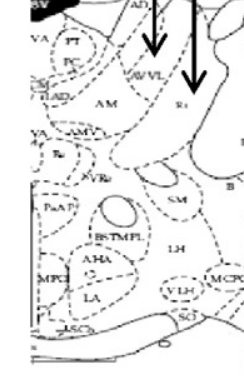

B

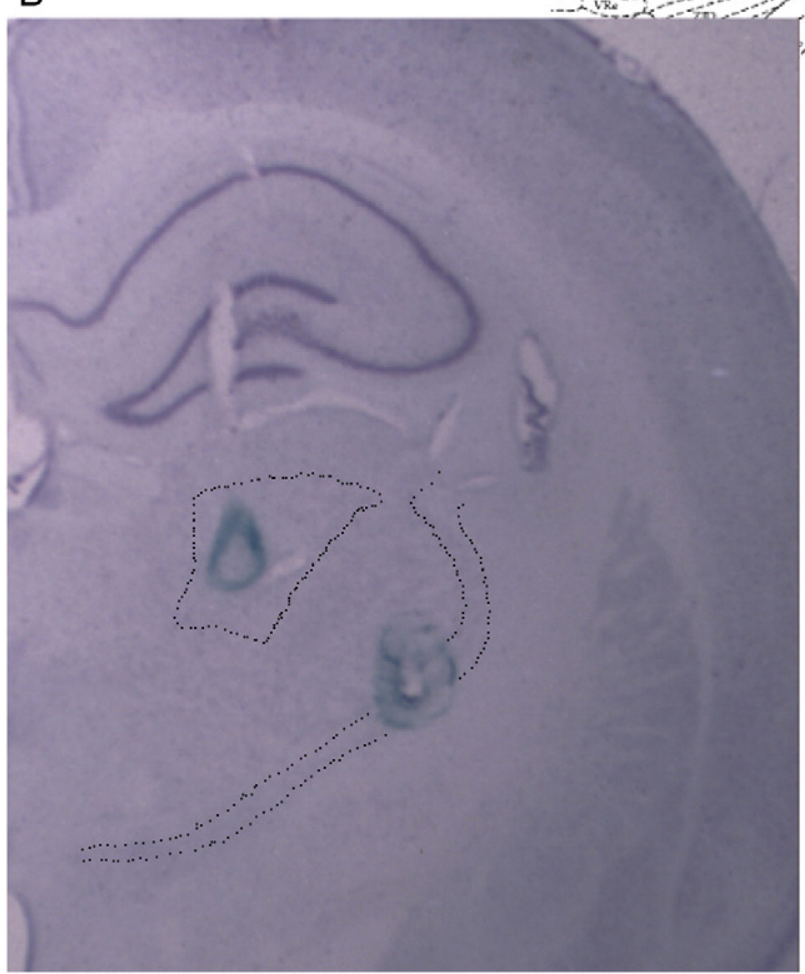

C

\begin{tabular}{|c|c|c|c|c|c|c|c|c|}
\hline & ctx 4 & ctx5 & ctx6 & ATN & Po & VPM & CRTN & rRTN \\
\hline Rat1 & $x$ & $x$ & $\mathrm{x}$ & $x$ & $x$ & $x$ & $x$ & $x$ \\
\hline Rat2 & $x$ & $x$ & $x$ & $x$ & $x$ & & $x$ & \\
\hline Rat3 & $x$ & $x$ & $x$ & $x$ & $x$ & & $x$ & $x$ \\
\hline Rat4 & $x$ & $x$ & $x$ & & $x$ & $x$ & $x$ & \\
\hline Rat5 & $x$ & $x$ & $x$ & & $x$ & $x$ & $x$ & $x$ \\
\hline Rat6 & $x$ & $x$ & $x$ & $x$ & $x$ & $x$ & $x$ & $x$ \\
\hline Rat7 & $x$ & $x$ & $x$ & $x$ & $x$ & $x$ & $x$ & $x$ \\
\hline Rat8 & $x$ & $x$ & $x$ & $x$ & & $x$ & & \\
\hline Rat9 & $x$ & $x$ & $x$ & $x$ & $x$ & $x$ & $x$ & $x$ \\
\hline Rat10 & $x$ & $x$ & $x$ & $x$ & & $x$ & & \\
\hline Rat40 & $x$ & $x$ & $\mathrm{x}$ & $x$ & $x$ & $x$ & $x$ & $x$ \\
\hline Rat13 & $x$ & $x$ & $x$ & $x$ & $x$ & $x$ & & $x$ \\
\hline Rat14 & $x$ & $x$ & $\mathrm{x}$ & & $\mathrm{x}$ & & $\mathrm{x}$ & $x$ \\
\hline Rat15 & $\mathrm{X}$ & $x$ & $x$ & $x$ & $x$ & $x$ & $x$ & \\
\hline Rat16 & $x$ & $x$ & $x$ & $x$ & $x$ & & $x$ & \\
\hline Rat17 & $x$ & $x$ & $x$ & & $x$ & $x$ & $x$ & \\
\hline
\end{tabular}

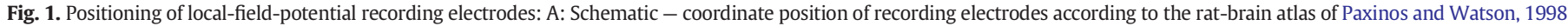

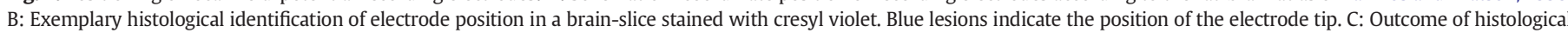

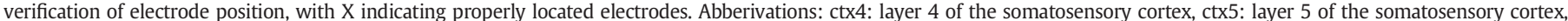

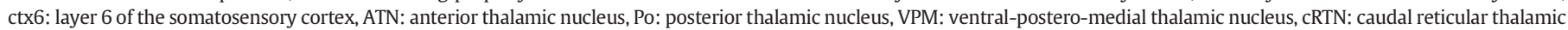
nucleus, rRTN: rostral reticular thalamic nucleus. (For interpretation of the references to color in this figure legend, the reader is referred to the web version of this article.) 
was described during SWD (Avoli et al., 1983; Pinault and O'Brien, 2005; Seidenbecher et al., 1998; Vergnes et al., 1989), but few studies investigated coherence during the periictal transition phase. In GAERS (genetic absence epileptic rats from Strasbourg) SWD emerge from 5 to $9 \mathrm{~Hz}$ sensory-motor like oscillations. These type of oscillations emerge from a desynchronized background EEG and show a medium to strong cortico-thalamic coherence, indicating a gradual process of neuronal synchronization accompanying SWD generation (Pinault and O'Brien, 2005; Pinault et al., 2001, 2006). On the other hand, these oscillations are not sufficient for the generation of SWD since non-epileptic control rats also possess these oscillations.

Sitnikova and van Luijtelaar (2006) compared unilateral, cortical, local, and long range intrahemispheric coherence with corticaltranshemispheric, unilateral intrathalamic, and unilateral corticothalamic coherence in genetic absence-epileptic WAG/Rij rats (Depaulis and van Luijtelaar, 2006; van Luijtelaar and Sitnikova, 2006) and reported that each network shows different frequency specific changes with SWD occurrence. The coherence analysis in this study, however, was restricted to a comparison $1 \mathrm{~s}$ prior as compared to $1 \mathrm{~s}$ following SWD onset whereas subsequent studies showed that this pre-ictal interval already contains SWD related changes in the form of spectral delta-theta precursor activity in frontal cortex and VPM (Sitnikova and van Luijtelaar, 2009; van Luijtelaar et al., 2011a). Moreover, cortico-thalamic coherence, thought to be most relevant for SWD occurrence (Depaulis and van Luijtelaar, 2006), was only investigated between frontal cortex and thalamus rather than between the more important SWD-instigator zone in the somatosensory cortex and its recipients, the VPM, RTN and Po (Meeren et al., 2002).

In the current study the dynamics of coherent neuronal network activity and spectral power changes in a longer transition from the pre-ictal to the ictal state are investigated in an extended part of the cortico-thalamo-cortical network. This enables us to sketch a potentially gradual developmental process of SWD, to investigate the relative contribution of different channel-pairs in SWD generation, and probably to detect (predictive) SWD precursor activity. For the spectral power, special attention is paid to the deep somatosensory cortex, where spectral precursor activity has not been studied before in this genetic model.

\section{Methods}

\section{Subjects}

16 male WAG/Rij rats, 6 to 9 months of age were used as experimental subjects. They were born and raised at the Department of Biological Psychology, Donders Centre for Cognition, Radboud University Nijmegen, The Netherlands. Prior to surgery rats were housed in pairs (High Makrolon ${ }^{\circledR}$ cages with Enviro Dri ${ }^{\circledR}$ bedding material and cage enrichment) with free access to food and water and were kept at a $12-12$ h light-dark cycle (lights off at $8.30 \mathrm{AM}$ ). After surgery rats were housed individually. The experiment was approved by the Ethical Committee on Animal Experimentation of Radboud University Nijmegen (RU-DEC). Efforts were made to keep the discomfort for the animals as minimal as possible.

\section{Surgery}

Implantation of the LFP recording electrodes was done in a stereotactic frame under isoflurane anesthesia. At the start of surgery, rats received a subcutaneous injection of the analgesic Rimadyl ${ }^{\circledR}$ and an intramuscular injection of atropine to prevent excessive salivary production. Body temperature was controlled and conserved via a heating pad. The local anesthetic Lidocaine was used on the incision points. Holes were drilled into the skull on top of the right hemisphere for the insertion of recording electrodes at the following positions: Somatosensory cortex: $\mathrm{A} / \mathrm{P}=0$, $\mathrm{M} / \mathrm{L}=-4.6$ depth $=-2.8$ (layer 4 ), -3.1 (layer 5), -3.6 (layer 6); anterior thalamus: $\mathrm{A} / \mathrm{P}=-1.4 \mathrm{M} / \mathrm{L}=-1$, depth $=-6.2$; rostral RTN: $\mathrm{A} / \mathrm{P}=-1.4, \mathrm{M} / \mathrm{L}=-1.9$, depth $=-6.6$; posterior thalamic nucleus: $\mathrm{A} / \mathrm{P}=-3.6, \mathrm{M} / \mathrm{L}=-2$, depth $=-5.4 ; \mathrm{VPM}: \mathrm{A} / \mathrm{P}=-4.16$, $\mathrm{M} / \mathrm{L}=-2.8$, depth $=-6$ and caudal RTN: $\mathrm{A} / \mathrm{P}=-3.1, \mathrm{M} / \mathrm{L}=-3.5$, depth $=-6.6$ (Fig. 1). All coordinates were determined relative to Bregma according to the rat-brain atlas of Paxinos and Watson (Paxinos and Watson, 1998). Electrode wires, assembled in a self-constructed electrode system (Lüttjohann and van Luijtelaar, 2012; van Luijtelaar et al., 2011b) were simultaneously inserted into the brain. Ground and reference electrodes were positioned epidurally on top of the cerebellum. The electrode assembly was fixed to the skull via dental cement. Postoperative analgesic Rimadyl ${ }^{\circledR}$ ( 24 and $48 \mathrm{~h}$ after surgery) was administered and rats were allowed to recover for two weeks.

\section{Recording of local field potentials}

Two weeks after surgery rats were placed individually in a $20 \times 35 \times 25 \mathrm{~cm}$ Plexiglas registration box and connected to the recording leads for multi-channel LFP recordings. These were attached to a swivel-contact, which allowed recording in freely moving animals. The LFP signals were amplified with a physiological amplifier (TD 90087, Radboud University Nijmegen, Electronic Research Group), filtered by a band pass filter with cut-off points at 1 (HP) and 100 (LP) and a $50 \mathrm{~Hz}$ Notch filter, and digitized with a constant sample rate of $2048 \mathrm{~Hz}$ by WINDAQ-recording-system (DATAQ-Instruments). Also the movements of the rat were registered by means of a Passive Infrared Registration system (PIR, RK2000DPC LuNAR PR Ceiling Mount, Rokonet). Each rat was recorded for a period of $4 \mathrm{~h}$ during the dark phase of the 12-12 LD cycle.

\section{Signal analysis}

A randomly chosen subset of 10 pre-ictal->ictal transition periods of $5 \mathrm{~s}$ duration were selected for each rat for signal analysis. As in an earlier study (Lüttjohann and van Luijtelaar (2012)), these 5-second epochs were centered around the occurrence of the first epileptic cortico-thalamic spike (FCTS). This is defined as a first sharp spike of at least twice the background LFPs, visible in all cortical and thalamic recordings, which is followed by rhythmic SWD activity (Fig. 2). The FCTS is considered to be the moment of "generalization" of epileptic activity from cortex to thalamus or equivalently the start of "full blown" cortico-thalamic SWD (Lüttjohann and van Luijtelaar, 2012).

Selected epochs of non-epileptic activity were used as control data. For each rat 10 epoch of 500 ms duration were randomly selected during passive wakefulness distant (by at least $5 \mathrm{~min}$ ) to SWD. Passive wakefulness is characterized by low amplitude, desynchronized, high frequency LFP signals recorded in a motionless rat. A major proportion of SWD tends to emerge during this state of vigilance (Drinkenburg et al., 1991).

Spectral properties (amplitude and phase) of LFP signals during transition and control periods were assessed via a time frequency analysis (TFA) using Hanning tapering. Since, for SWD, changes in connectivity are known to occur within timeframes as short as $500 \mathrm{~ms}$ (directional coupling from cortex to thalamus was found only during the first 500 ms of SWDs (Meeren et al., 2002)), assessment of spectral power in this study was also performed in $500 \mathrm{~ms}$ timeframes shifting along the pre-ictal->ictal transition period in steps of $50 \mathrm{~ms}$. Given the size of the analysis window, frequency resolution was restricted to an accuracy of $2 \mathrm{~Hz}$. TFA was performed for the frequency-range 2 to $60 \mathrm{~Hz}$.

Coherent neuronal network activity (network communication) was quantified with pairwise-phase consistency analysis (PPC) 

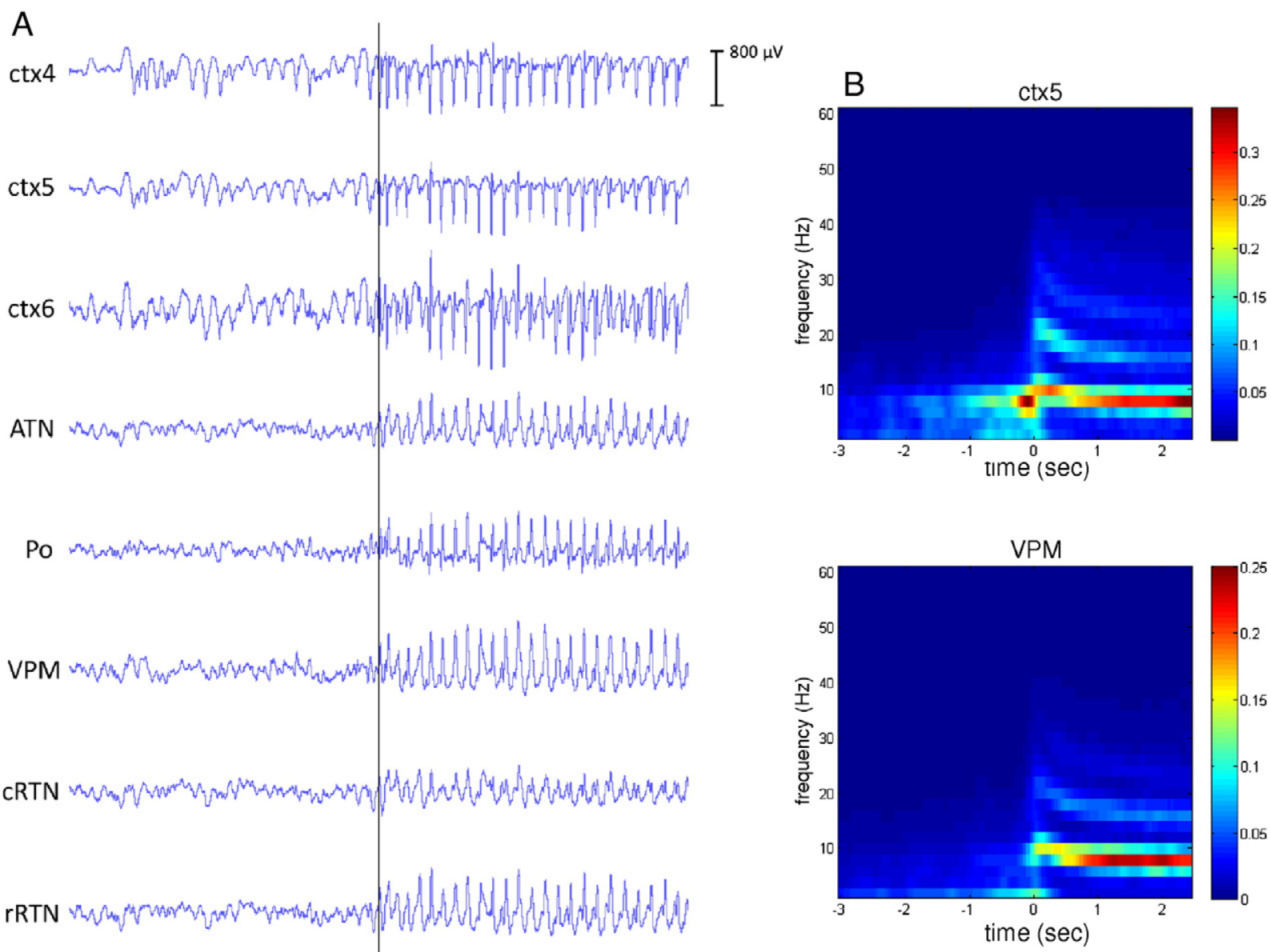

C Pattern of strongest SWD precursor activity across brain structures. Frequency of occurrence is given in $\%$ of total.

\begin{tabular}{|c|c|c|c|}
\hline brainstructure & $\begin{array}{c}\text { individual } \\
\text { delta- } \\
\text { precursor }\end{array}$ & $\begin{array}{c}\text { individual } \\
\text { theta- } \\
\text { precursor }\end{array}$ & $\begin{array}{c}\text { combined } \\
\text { delta/theta } \\
\text { precursor }\end{array}$ \\
\hline ctx 4 & 10.8 & 15.5 & 63.4 \\
ctx 5 & 7.7 & 11.5 & 75.6 \\
ctx 6 & 7.0 & 49.4 & 41.0 \\
P0 & 53.2 & 7.5 & 23.7 \\
VPM & 54.7 & 4.1 & 25.0 \\
ATN & 33.6 & 30.5 & 17.9 \\
cRTN & 14.1 & 26.7 & 44.4 \\
rRTN & 18.4 & 18.4 & 19.7 \\
\hline
\end{tabular}

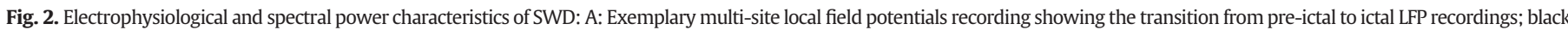

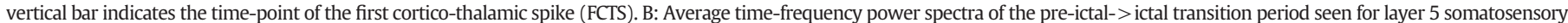

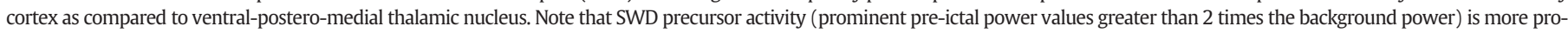

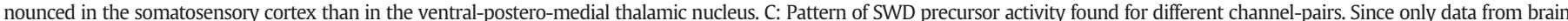

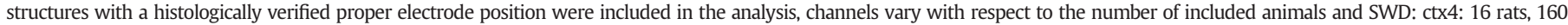

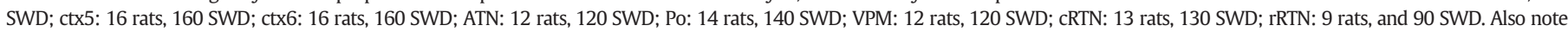

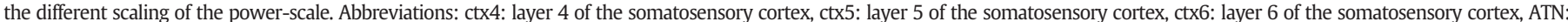
anterior thalamic nucleus, Po: posterior thalamic nucleus, VPM: ventral-postero-medial thalamic nucleus, cRTN: caudal reticular thalamic nucleus, rRTN: rostral reticular thalamic nucleus.

(Vinck et al., 2010). The PPC metric quantifies the consistency of the phase difference between two signals, across observations (SWD). It is a normalized measure, and it is bounded between 0 (signifying the lack of synchronization) and 1 (signifying perfect synchronization). In these respects the PPC metric resembles the well-known coherence coefficient. However, the coherence coefficient is a metric that is biased, where the size of the bias depends on the number of observations. This is not the case with the PPC metric, which makes it particularly suitable in a setting where the number of observations
(SWD) is relatively low (and consequently the size of the estimation bias in the coherence is high).

This difference in bias is due to a difference in the calculation of the estimates:

Both coherence and PPC are calculated from the single trial cross-spectral densities, which quantify the phase difference of oscillations between a pair of signals at a given frequency. The cross-spectral density can be visualized in a 2-dimensional Cartesian 
plane as a vector from the origin, where the angle relative to the positive X-axis represents the phase difference. The coherence is computed from the length of the average cross-spectral density vector. This average is obtained by putting all single-trial cross-spectral density vectors head to tail, and dividing the resulting vector by the number of observations. In the presence of true phase synchronization (i.e. a consistent phase difference across observations), the single trial cross-spectral density vectors have a tendency to point in the same direction, yielding a large average cross-spectral density vector, and hence a large coherence coefficient. In the absence of true phase synchronization (i.e. the cross-spectral density vectors are pointing into random directions), the average crossspectral density vector will be much smaller, but will always have a non-zero value. Therefore, when trying to estimate the true coherence (which is 0 in the absence of phase synchronization) from a finite number of observations, the estimate will always be too high (hence biased). Moreover, the size of the bias will be inversely related to the number of observations, because one gets 'closer to zero' with the average of the cross-spectral densities when there are more observations.

The PPC is computed from the average angular distance between all pairs of cross-spectral density vectors. This means that for each pair of observations, the difference between the estimated phase difference is computed (yielding the angular distance), and the cosine of the angular distances is averaged across all pairs of observations. In the presence of true phase synchronization, the single-trial cross-spectral density vectors point into the same direction, and the pairwise angular distances are small (i.e. close to 0 , and hence with a cosine close to 1 ), yielding a large PPC value. In the absence of true phase synchronization, the single-trial cross-spectral density vectors point into random directions, yielding a distribution of pairwise angular distances between 0 and $180^{\circ}$, with cosines between 1 and -1 , yielding a small PPC value, because the negative cosines cancel the positive cosines in the average. It was shown that this metric is not (or much less) biased by the number of observations (Vinck et al., 2010). For more detailed information on the PPC and coherence analysis and their bias properties the reader is referred to Vinck et al. (2010) and Maris et al. (2007).

PPC data as well as spectral power during pre-ictal and ictal periods were compared to control periods with the aid of a non-parametric cluster based permutation test (Maris and Oostenveld, 2007). This test has been shown to be a reliable statistical method for the analysis of neurophysiological data requiring comparison along multiple time and frequency bins, with efficient control of type II errors (Maris et al., 2007). Basically the following steps are performed:

1) First the datasets of condition 1 (transition period) and condition 2 (control period) are compared by Students' $t$-test. All datapoints (time-frequency points) reaching significant difference are grouped to a cluster based on temporal adjacency. For each cluster the sum of the T-values (test-statistic of the previous $t$-test) is calculated.

2) Next, the original time-frequency data-sets are randomly distributed to either condition 1 or 2 . By this the difference between control and transition period is destroyed.

3) For each random distribution (in our case 500) step 1 is performed.

4) Lastly the summed t-values of a cluster (calculated in step 1) for the real data-set and the random distributions are displayed in a histogram.

In case the summed t-value computed from the real dataset is positioned below the 2.5-th or above the 97.5-th quantile of the histogram ( $p<0.05$ two-sided), the real dataset deviates (with a certainty of 95\%) from randomized datasets, which do not have a difference between control and transition period. The real cluster can thus be considered as representing a significant difference between control and transition period.

In addition to the permutation test, the percentage of pre-ictal periods showing power increases more than 2-times the background power within the delta and theta frequency range were registered for time frequency data. This operating scheme has been used earlier to quantify spectral SWD precursor activity (van Luijtelaar et al. (2011a)).

All statistical and signal analyses were performed with FieldTrip, an open-source Matlab-based toolbox for advanced analysis of e.g. electrophysiological data (Oostenveld et al., 2011).

Only data from brain structures with a histological verified proper electrode position were included in the statistical analysis (Fig. 1). To facilitate the finding of the location of the tip of the recording electrodes, a direct current ( $9 \mathrm{~V}, 25 \mu \mathrm{A}, 10 \mathrm{~s}$ duration) was passed through each electrode in the deeply anesthetized rat at the end of the experiment. Next, rats were perfused with a potassiumferrocyanide-formaldehydephosphate solution, coloring these lesions at the end of each electrode tip. Brains were fixed in a $30 \%$ sucrose solution, $0.1 \mathrm{ml}$ PBS, cut in $40 \mu \mathrm{m}$ coronal slices with the aid of a microtome, and stained with Cresyl violet. Only electrodes for which the midpoint of the small lesion was located within the target structure were considered properly implanted and included in statistical analysis (Fig. 1).

\section{Results}

Electrophysiological and spectral characteristics: the presence of precursor activities

All rats showed SWD: mean 10 per hour, mean duration SWD $7 \mathrm{~s}$. Rhythmic spike and wave activity in the typical frequency of 8-10 Hz was present in all recorded channels (Fig. 2a). Time-frequency spectra of all recorded channels showed highest power values in the SWD characteristic frequency band of 8-10 Hz during the ictal period (Fig. 2b). Frequency modulation from 10 to $12 \mathrm{~Hz}$ at the beginning of the SWD, quickly followed (within $1 \mathrm{~s}$ ) by oscillations in the $7-8 \mathrm{~Hz}$ band was noticed (Bosnyakova et al., 2006). Somewhat less intense maxima in power were seen at the first (around $20 \mathrm{~Hz}$ ) and sometimes second harmonic (around $30 \mathrm{~Hz}$ ).

Non-parametric cluster based permutation test revealed that all channels showed a significant increase in power in the delta $(2-4 \mathrm{~Hz})$ and theta $(6-12 \mathrm{~Hz})$ frequency range as compared to the control period (all p's $<0.005$ ) during the pre-ictal interval ( $2.5 \mathrm{~s}$ prior to FCTS until FCTS). Channels, however, differed regarding their pattern of maximal pre-ictal power values (increases in power larger than 2-times the background power): Earliest and most pronounced pre-ictal power was noticed for the deep layers of the somatosensory cortex (all 16 rat, 160 $S W D$ ), with ctx5 showing delta and theta precursor activity simultaneously in $75 \%$ of pre-ictal periods. These started on average $2 \mathrm{~s}$ prior to FCTS (Fig. 2b). In thalamic recordings, on the other hand, maximal power values started at $0.75 \mathrm{~s}$ (range 0.25 to $1 \mathrm{~s}$ ) prior to FCTS. Whereas for the cRTN (13 rats, 130 SWD) combined delta-theta precursor was most common (44.4\% of SWD), VPM (12 rats, 120SWD) and Po (14 rats, 140 SWD) precursor activity was most often (54.7 and 53.2\% of SWD) seen to be restricted to the delta band (see Fig. $2 \mathrm{c}$ for more details).

\section{PPC during the pre-ictal period}

Four of the twenty-eight channel-pairs showed significant changes in PPC (Fig. 3, Table 1) during the pre-ictal period. Interestingly, in all cases a reduction compared to non-epileptic control periods was found and in three out of these four pairs the caudal reticular thalamic nucleus was involved:

\footnotetext{
- At $1.2 \mathrm{~s}$ prior to FCTS, channel-pair cRTN-ctx4 (13 rats, 130 SWD) showed a significant reduction of $0.2(\mathrm{p}<0.025)$. PPC reduced from 0.35 (control) to 0.15 for frequencies around $20 \mathrm{~Hz}$.
} 

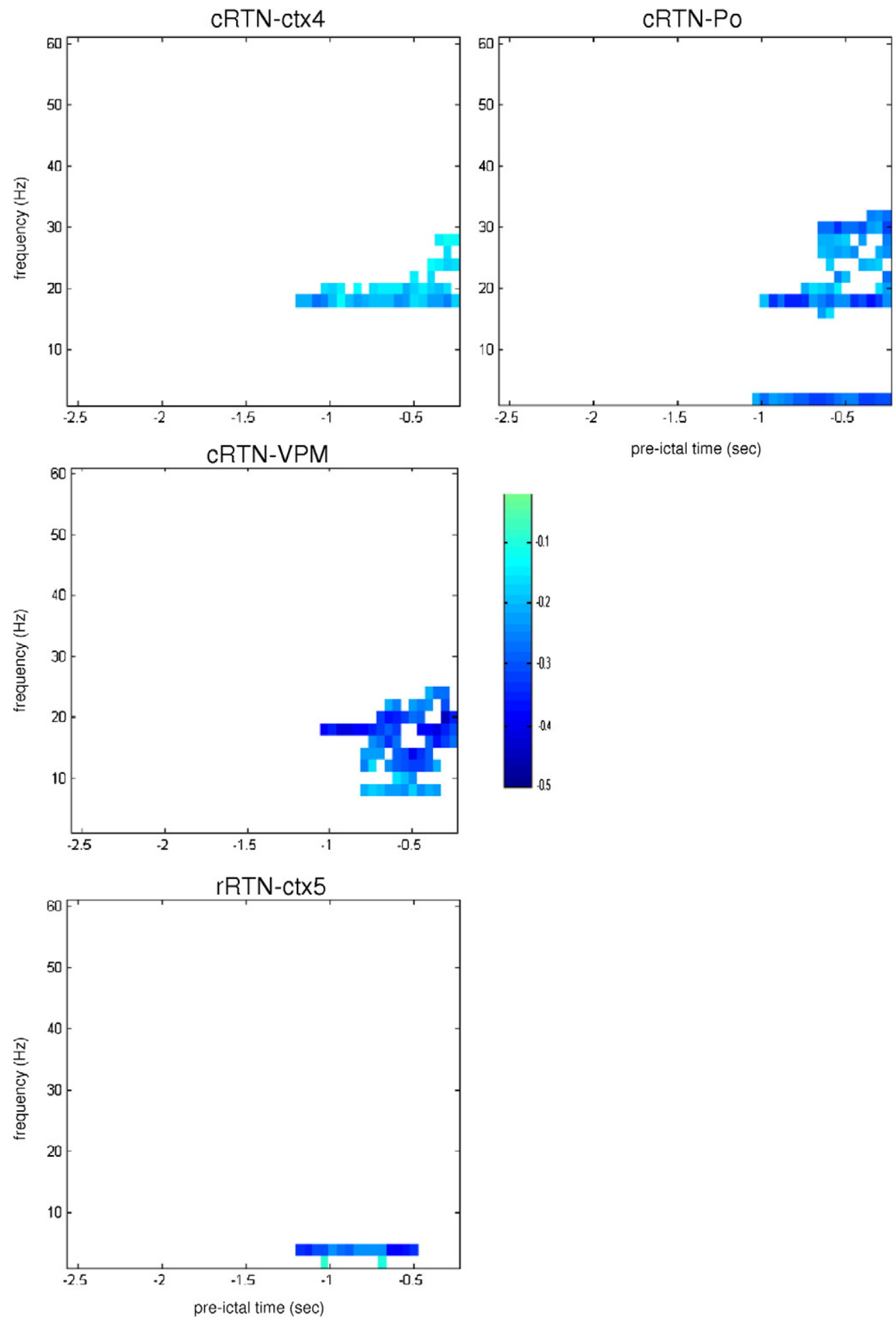

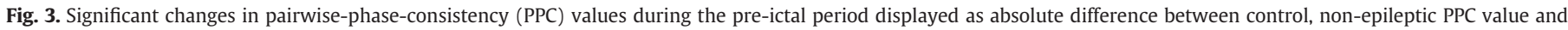

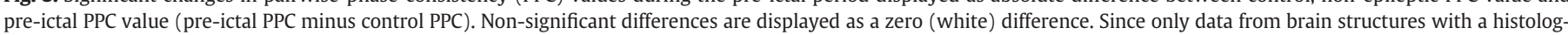

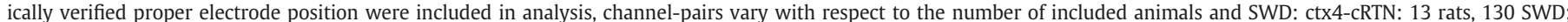

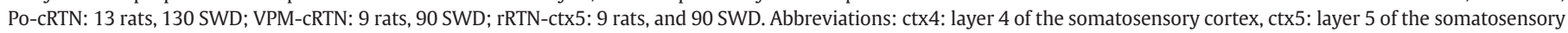
cortex, Po: posterior thalamic nucleus, VPM: ventral-postero-medial thalamic nucleus, cRTN: caudal reticular thalamic nucleus, rRTN: rostral reticular thalamic nucleus.

- Shortly thereafter (about $1 \mathrm{~s}$ prior to FCTS) a reduction from 0.5 (control) to 0.25 was found for channel-pair cRTN-Po (13 rats, 130 SWD $)$ at the $20-30 \mathrm{~Hz}(\mathrm{p}<0.025)$ and of 0.3 around $2 \mathrm{~Hz}$ $(\mathrm{p}<0.025)$.

- The third channel-pair showing a significant pre-ictal reduction was cRTN-VPM (9 rats, 90 SWD). For this pair, decreases were found in high frequencies but also in the SWD characteristic $10 \mathrm{~Hz}$ band. At
$10-12 \mathrm{~Hz}$ a reduction of 0.22 (from 0.6 control to 0.38 pre-ictal) was seen $0.8 \mathrm{~s}$ prior to FCTS $(\mathrm{p}<0.025)$. At $14-20 \mathrm{~Hz}$ this channel-pair showed a reduction of $0.35(\mathrm{p}<0.025)$.

- Lastly, channel-pair rRTN-ctx5 (9 rats, 90 SWD) showed a significant decrease in pre-ictal PPC at $1.2 \mathrm{~s}$ prior to FCTS. PPC values dropped by 0.15 from 0.52 (control) to 0.37 ( $p<0.01$ ). This decrease was seen around $4 \mathrm{~Hz}$. 
Table 1

Overview of significant changes in PPC per channel pair: indicated is either a significant increase $(\uparrow)$ or decrease $(\downarrow)$ in the pre-ictal and ictal period as compared to control period, and the frequencies for which this change is found. For details on the exact timing (onset and stop of changes see main text). Abbreviations: ctx4: layer 4 of the somatosensory cortex, ctx5: layer 5 of the somatosensory cortex, ctx6: layer 6 of the somatosensory cortex, ATN: anterior thalamic nucleus, Po: posterior thalamic nucleus, VPM: ventral-postero-medial thalamic nucleus, cRTN: caudal reticular thalamic nucleus, rRTN: rostral reticular thalamic nucleus.

\begin{tabular}{|c|c|c|c|}
\hline Channel-pair & $\begin{array}{l}\text { No. of rats/ } \\
\text { SWD }\end{array}$ & Pre-ictal & Ictal \\
\hline ctx4-Po & 14,140 & & $\uparrow 8-48 \mathrm{~Hz}$ \\
\hline ctx5-Po & 14,140 & & $\uparrow 8-48 \mathrm{~Hz}$ \\
\hline ctx6-Po & 14,140 & & $\uparrow 8-48 \mathrm{~Hz}$ \\
\hline ctx4-VPM & 12,120 & & $\uparrow 8-12 \mathrm{~Hz}$ and harmonics \\
\hline ctx5-VPM & 12,120 & & $\uparrow 8-12 \mathrm{~Hz}$ and harmonics \\
\hline ctx6-VPM & 12,120 & & $\uparrow 8-12 \mathrm{~Hz}$ and harmonics \\
\hline ctx4-ATN & 12,120 & & $\uparrow 8-12 \mathrm{~Hz}$ and harmonics \\
\hline ctx5-ATN & 12,120 & & $\uparrow 8-12 \mathrm{~Hz}$ and harmonics \\
\hline ctx6-ATN & 12,120 & & $\uparrow 8-12 \mathrm{~Hz}$ and harmonics \\
\hline cRTN-ctx4 & 13,130 & $\downarrow 20 \mathrm{~Hz}$ & Back to baseline level \\
\hline cRTN-ctx 5 & 13,130 & & Late (at $1.5 \mathrm{~s}$ ) $\uparrow 10-12 \mathrm{~Hz}$ \\
\hline cRTN-ctx6 & 13,130 & & Late (at $1 \mathrm{~s}) \uparrow 10-12 \mathrm{~Hz}$ \\
\hline rRTN-ctx4 & 9,90 & & $\uparrow$ around $20 \mathrm{~Hz}$ \\
\hline rRTN-ctx5 & 9,90 & $\downarrow 4 \mathrm{~Hz}$ & $\begin{array}{l}\uparrow \text { around } 20 \mathrm{~Hz} \text {; at } 4 \mathrm{~Hz} \text { back } \\
\text { to baseline level }\end{array}$ \\
\hline rRTN-ctx6 & 9,90 & & $\uparrow$ around $20 \mathrm{~Hz}$ \\
\hline cRTN-rRTN & 8,80 & & Late $(2 \mathrm{~s}) \uparrow 8-10 \mathrm{~Hz}$ \\
\hline cRTN-ATN & 9,90 & & \\
\hline cRTN-VPM & 9,90 & $\begin{array}{l}\downarrow 10-12 \mathrm{~Hz} \text { and } \\
\downarrow 14-20 \mathrm{~Hz}\end{array}$ & $\begin{array}{l}10-16 \mathrm{~Hz} \text { back to baseline; } 20 \mathrm{~Hz} \\
\text { still } \downarrow ; \text { new } \downarrow \text { at } 30-40 \mathrm{~Hz}\end{array}$ \\
\hline cRTN-Po & 13,130 & $\begin{array}{l}\downarrow 20-30 \mathrm{~Hz} \\
\text { and } 2 \mathrm{~Hz}\end{array}$ & $\begin{array}{l}\uparrow 8-10 \mathrm{~Hz} ; 2,20-30 \mathrm{~Hz} \text { back to } \\
\text { control; new } \downarrow \text { at } 30-46 \mathrm{~Hz}\end{array}$ \\
\hline ATN-Po & 10,100 & & $\uparrow 8-10 \mathrm{~Hz}$ \\
\hline rRTN-Po & 9,90 & & $\uparrow 10-12 \mathrm{~Hz}$ and harmonic \\
\hline VPM-Po & 10,100 & & \\
\hline VPM-ATN & 9,90 & & \\
\hline VPM-rRTN & 7,70 & & \\
\hline ATN-rRTN & 7,70 & & Late $(0.5 \mathrm{~s}) \uparrow 8-10 \mathrm{~Hz}$ \\
\hline $\operatorname{ctx} 4-\operatorname{ctx} 5$ & 16,160 & & $\uparrow 8-48 \mathrm{~Hz}$ \\
\hline $\operatorname{ctx} 4-\operatorname{ctx} 6$ & 16,160 & & $\uparrow 8-48 \mathrm{~Hz}$ \\
\hline $\operatorname{ctx} 5-\operatorname{ctx} 6$ & 16,160 & & $\uparrow 8-48 \mathrm{~Hz}$ \\
\hline
\end{tabular}

\section{PPC during the ictal period}

Most channel-pairs showed increases in PPC at the transition to the ictal period. This transition took place in a strong and rather abrupt fashion and was found to be channel-pair and frequency specific (Table 1):

Cortico-thalamic channel-pairs:

Earliest increases in PPC were found between all three layers of the somatosensory cortex and the posterior thalamic nucleus (all channel pairs 14 rats, 140 SWD). They started to occur $0.2 \mathrm{~s}$ prior to FCTS and lasted until the end of the analysis window ( $2.5 \mathrm{~s}$ following FCTS) (all p's $<0.01$ ). These early increases in PPC between cortex and Po were quickly followed by increases in PPC in channel-pairs ctx4-VPM, ctx5-VPM, ctx6-VPM, ctx4-ATN, ctx5-ATN and ctx6-ATN (all 12 rats, 120 SWD). For these channel-pairs, significantly higher PPC values as compared to control periods were reached between $0.15 \mathrm{~s}$ to $0.05 \mathrm{~s}$ prior to FCTS, depending on frequency (all p's $<0.01$ ), and also lasted until the end of the analysis window (2.5 s following FCTS). PPC increases of cortex-ATN and cortexVPM channel-pairs were restricted to $10-12 \mathrm{~Hz}$ and its two harmonics. Cortex-Po channel-pairs, on the other hand, demonstrated PPC increases in a much more extended frequency range spanning from 8 to $48 \mathrm{~Hz}$ (Fig. 4). The average absolute increase in PPC for these channel-pairs ranged from 0.3 to 0.65 .

The two cortico-thalamic channel-pairs cRTN-ctx4 (13 rats 120 SWD) and rRTN-ctx5 (9 rats, 90 SWD), for which a decrease in PPC was seen in the pre-ictal interval, did not show significantly decreased PPCs in the ictal-period anymore. For both channel-pairs the decreased PPC values returned to control values, at $0.1 \mathrm{~s}$ and $0.45 \mathrm{~s}$ prior to FCTS respectively. Caudal RTN-ctx 4 remained at its baseline level during the complete ictal period and did not show any increases in PPC during the ictal interval; rRTN-ctx5, by contrast, showed a significant increase in PPC around $20 \mathrm{~Hz}$ starting $0.1 \mathrm{~s}$ prior to FCTS $(\mathrm{p}<0.001)$. The same was found for channel-pair rRTN-ctx 4 and rRTN-ctx 6 ( 9 rats, 90 SWD and p's $<0.02$ for both). Similar to channel-pair cRTN-ctx4, channel-pairs cRTN-ctx5 and cRTN-ctx6 (all 13 rats, 130 SWD) also showed baseline PPC levels at the beginning of the ictal period. Only towards the end of the ictal period did both channel-pairs show an increase in PPC values in the SWD characteristic $10-12 \mathrm{~Hz}$ band, starting 1.5 and $1 \mathrm{~s}$ after FCTS respectively (both p's $<0.025$ ).

Intra-thalamic channel-pairs:

The two intra-thalamic channel-pairs that already showed a decrease in PPC in the pre-ictal period again showed changes in PPC when entering the ictal period:

Channel-pair cRTN-VPM ( 9 rats, 90 SWD) returned from its decreased pre-ictal PPC value to baseline levels for the $10-16 \mathrm{~Hz}$ band, while keeping its decreased pre-ictal PPC values at $20 \mathrm{~Hz}$. In addition, a decrease from 0.6 (control) to 0.25 (ictal) was found for 30 to $40 \mathrm{~Hz}$ (all p's $<0.025$ ).

Channel-pair cRTN-Po (13 rats, 130 SWD) also returned its decreased pre-ictal PPC values for $20-30 \mathrm{~Hz}$ towards control, and switched towards decreased PPC values (baseline 0.4 to ictal $=0.2)$ for the higher $(30-46 \mathrm{~Hz})$ frequencies $(p<0.01)$. However, this channel-pair also suddenly showed increases in PPC ( 0.5 control to $0.8 \mathrm{ictal}$ ) in the $8-10 \mathrm{~Hz}$ band starting at $0.035 \mathrm{~s}$ prior to FCTS $(\mathrm{p}<0.01)$.

Besides the cRTN, ATN and rRTN also showed an increase in PPC with the Po (ATN-Po: 10 rats, 100 SWD; rRTN-Po: 9 rats, 90 $S W D$ ) (8-12 Hz band, all p's $<0.01$ ) (Fig. 5). The only thalamic nucleus that did not show an increase in PPC with Po was the VPM (VPM-Po: 10 rats, 100 SWD). During the ictal period the VPM only showed increased PPC values with the three layers of the somatosensory cortex (all 12 rats, 120 SWD) (p's $<0.025$ ), but not with any other thalamic nucleus (all p's $>0.05$ ) (Fig. 5).

Lastly, ATN-rRTN ( 7 rats, 70 SWD) and rRTN-cRTN ( 8 rats, 80 SWD) showed increased PPC values $(8-10 \mathrm{~Hz})$ that started to occur at $0.5 \mathrm{~s}$ and $2 \mathrm{~s}$ after FCTS respectively (p's $<0.025$ ). No changes in PPC were found for channel pair cRTN-ATN ( 9 rats, 90 SWD) $(\mathrm{p}>0.05)$.

\section{Cortico-cortical channel-pairs:}

All intra-cortical channel-pairs (all 16 rats, 160 SWD) showed abrupt increases in PPC, from control values of around 0.7 to 0.9 ictally, within the frequency range of 8 to $48 \mathrm{~Hz}$ (all p's $<0.01$ ). Significant changes were seen somewhat earlier for the higher frequencies ( $0.1 \mathrm{~s}$ prior to FCTS, $20-48 \mathrm{~Hz}$ ) as compared to lower frequencies $(8-20 \mathrm{~Hz})$. Increased PPC values were maintained until the end of the analysis window ( $2.5 \mathrm{~s}$ following FCTS).

\section{Discussion}

The study of network interaction has the potential to unravel crucial changes in the communication between brain structures, which underlie/go along with the generation of generalized absence seizures. In the current study, dynamics of spectral power and phase characteristics were described for pre-ictal->ictal transition periods within an extended part of the cortico-thalamo-cortical system of absence epileptic WAG/Rij rats, including the deep layers of the perioral-region of the somatosensory-cortex (the local instigator zone of SWDs) and several important (based on anatomical, lesion, 

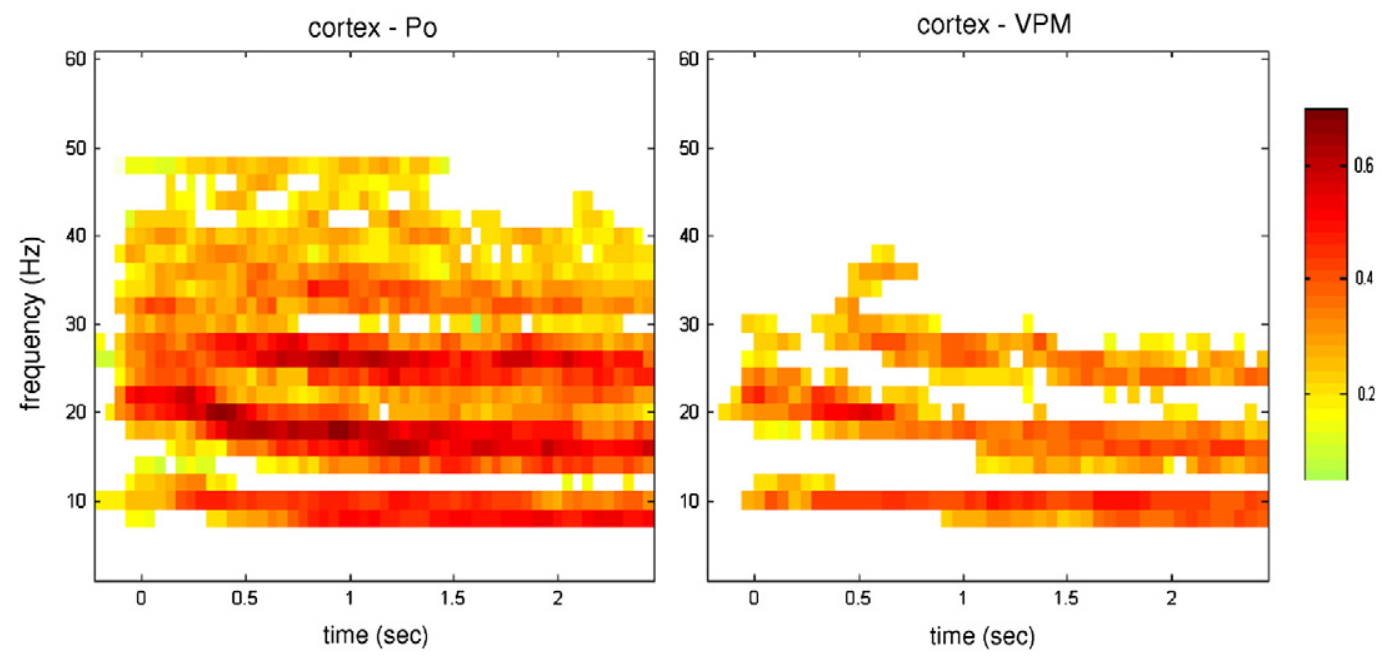

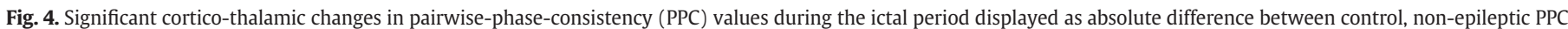

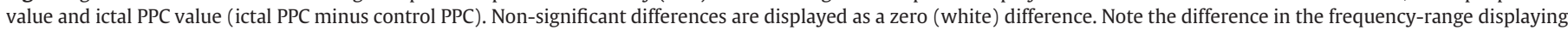

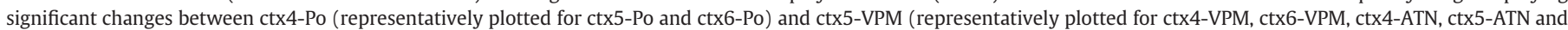

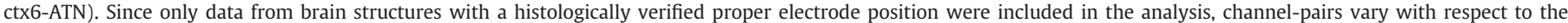

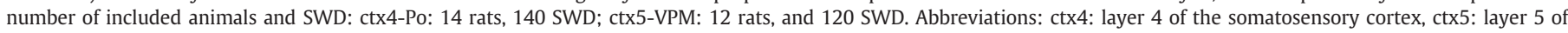

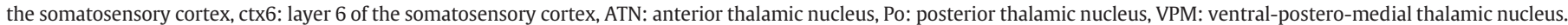

neurophysiological and modeling studies) thalamic nuclei. PPC analysis (Vinck et al., 2010) was used to study the role of coherent neuronal network activity, a proposed mechanism of neuronal communication (Buzsaki, 2006; Schnitzler and Gross, 2005), in the generation of SWD. Time-frequency analysis was used to study changes in spectral power properties and to assess the presence of SWD precursor activity. For the latter analysis, special attention was paid to the somatosensory cortex (SWD instigator zone), where spectral precursor activity was not studied before in this genetic model. The combination of both analyses enabled us to draw the following conclusions regarding the generation of SWD:

Network communication during the pre-ictal period: decoupling of the caudal reticular thalamic nucleus

A remarkable result of the current study was that the pre-ictal period was characterized by decreases in PPC, it was found in 4 out of 28 channel-pairs. Here, the cRTN is especially noteworthy: it decouples from Po, VPM and ctx4.

A pre-ictal decrease in (long-range) synchrony as compared to interictal periods was also reported in patients with partial seizures and absence seizures, which was seen several seconds prior to SWD onset (Amor et al., 2009; Le Van Quyen, 2005; Mormann et al., 2003a,b). These authors gave three different suggestions on how to interpret such a pre-ictal desynchronization: 1) desynchronization might provide an 'idle' population of neurons, that can be recruited into the epileptic process more easily; 2) desynchronization might reflect a depression of synaptic inhibition and 3) desynchronization might isolate the epileptic focus from regulatory influences of other brain structures and thereby facilitate local pathological recruitment (Le Van Quyen, 2005).

The decoupling of the caudal RTN found in the current study provides support for the first and second suggestion: The reticular thalamic nucleus is known to strongly regulate the activity of thalamo-cortical and cortico-thalamic relay cells via its GABAergic projections, and is often regarded/described as an attentional gate or spotlight (Coenen, 1995; Crick, 1984; Guillery et al., 1998; Pinault, 2004). It might be that only when the inhibitory regulation $\mathrm{f}$ the cRTN onto the thalamo-cortical system is gone (disinhibition), the local cortical instigator zone has the chance to entrain the thalamus into SWD activity. The decoupling from the Po might especially be seen as crucial. This nucleus has been proposed to be strongly involved in the generation of SWD given early and strong increases in coupling (as measured with non-linear association analysis) with the cortical focus (Lüttjohann and van Luijtelaar, 2012).

Less evidence is provided for the third possibility; which would imply a gradual increase in local cortical PPC indicating a local preparation/recruitment towards enhanced excitability, which is only possible when the cortical zone is isolated from surrounding influences. Such a state has been reported in the human condition several hundred milliseconds prior to SWD onset in whole head MEG studies (Amor et al., 2009; Gupta et al., 2011). A local preparation state, as found in patients, might not be necessary but cannot be excluded. Electrical evoked potential studies in this model demonstrated that the deep layers of the somatosensory cortex show an increased excitability as compared to motor cortex and healthy control animals independent of alertness (Lüttjohann et al., 2011). However, it is not known whether the local excitability changes in the pre-ictal period, as would have been predicted by interpretation possibility 3; single trial evoked potentials preceding the onset of SWD might be an option to investigate putative dynamics of cortical excitability.

Next to the three interpretation possibilities proposed in human studies (see above) we want to propose a fourth possibility on how decoupling of the caudal RTN might facilitate SWD occurrence: Decoupling of the caudal RTN might change the way in which external stimuli reach the hyperexcitable somatosensory cortex. Such external inputs are imperative for SWD occurrence: Abbasova et al. (2010) accomplish a complete abolishment of SWD by pharmacological blockage of the peripheral trigeminal nerve. Therefore, decoupling of the cRTN might change trigeminal input into an effective trigger for a somatosensory 'hyperdischarge'. Especially the decoupling of the cRTN from cortical input layer 4 and the VPM, which is the primary relay nucleus of incoming somatosensory stimuli, might be in favor of this latter interpretation since disinhibition allows a better transfer of information through the thalamus. 

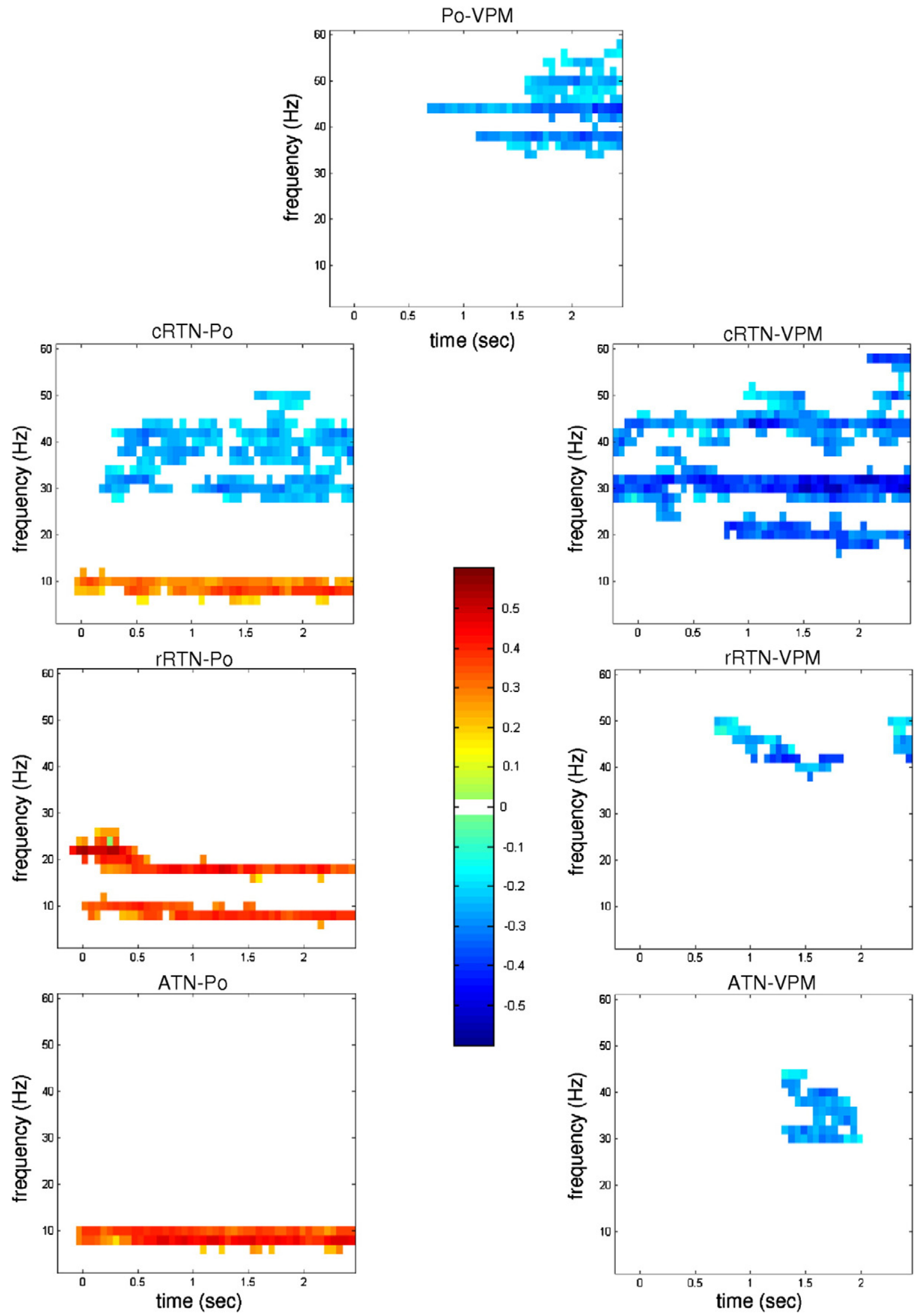

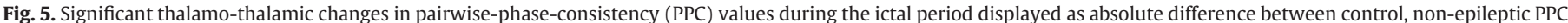

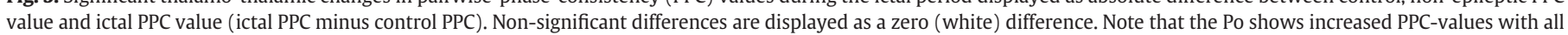

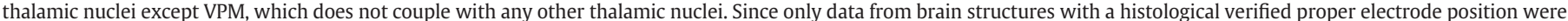

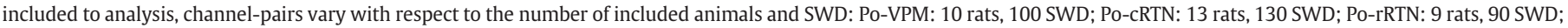

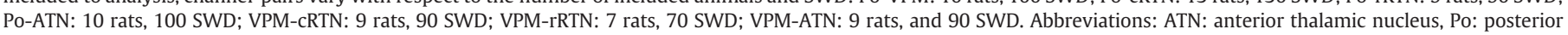
thalamic nucleus, VPM: ventral-postero-medial thalamic nucleus, cRTN: caudal reticular thalamic nucleus, rRTN: rostral reticular thalamic nucleus. 
Independent of the four different possibilities of interpretation, we propose that the decoupling of the cRTN might be a prerequisite for SWD occurrence; it prepares the cortico-thalamo cortical network towards a pro-epileptic state. Earlier pharmacological studies by Aker et al. (2006), and a lesion study by Meeren et al. (2009), demonstrating an increase in SWD activity after deactivation of the cRTN, which might be seen as an artificially created decoupling of the cRTN from all other thalamic nuclei, strongly support this conclusion.

In the same line of reasoning, it would be interesting to investigate whether the cRTN is also involved in the disruption or termination of an ongoing SWD. Based on the above proposed role of the cRTN, as having a dragging effect towards epileptic oscillations, one might expect an increase in PPC between cRTN and cortex towards the end of the SWD. The late ictal increase between cRTN and ctx5/6 found in the current study as well as earlier work in slices demonstrating that the RTN is involved in the regulation of SWD duration (Sohal et al., 2003) are in line with this idea. In addition, in in vivo experiments changes in the intercellular communication within the RTN via gap junctional coupling were shown to influence SWD duration (Proulx et al., 2006). Detailed network investigations focusing on the end of SWD might further enlarge our knowledge on this topic.

It is theoretically possible that parts of the pre-ictal decreases in PPC can be attributed to changes in vigilance, given the preference of SWD to occur in an intermediate level of alertness (Drinkenburg et al., 1991; Smyk et al., 2011). It is, however, more likely that the decoupling of the cRTN is a phenomenon that is specific to SWD generation. In favor of this argument is the channel-pair specificity of PPC decreases (vigilance related changes might be less specific). Also, the subtle increases in power (see the Electrophysiological and spectral characteristics: the presence of precursor activities section) and their temporal proximity to the FCTS are in favor of our view that they are SWD related and not vigilance related. In addition, the similar pre-ictal desynchronization in humans could even be used for automated seizure prediction with a specificity up to $100 \%$ (Mormann et al., 2003a,b).

There was no clear increase in PPC between cortex and thalamus in the 2-3 s pre-ictally; strong increases in phase synchronization in the SWD's frequency band and its harmonics occurred $100 \mathrm{~ms}$ before FCTS. Results from the GAERS model of absence epilepsy, showing medium to strong cortico-thalamic coherence during their SWD preceding 5-9 Hz oscillations (Pinault and O'Brien, 2005), suggest that the cortico-thalamo-cortical system also prepares toward a pro-epileptic state by facilitating and alleviating network communication between epileptic focus and given thalamic counterparts. Non-linear association analyses (amplitude coupling) of cortico-cortical network in WAG/Rij rats also revealed gradual changes preceding the onset of FCTS by about $1 \mathrm{~s}$ (Meeren et al., 2002). Likewise, a pre-ictal increase in amplitude coupling between cortical focus and the posterior thalamic nucleus, together with changes in time delays (Lüttjohann and van Luijtelaar, 2012), hinted towards a gradual increase in cortico-thalamic network communication prior to SWD onset. Apparently these increases in cortico-thalamic and thalamo-cortical coupling in WAG/Rij rats are not established via linear, stable, phase relationship between signals - but might be of non-linear nature (which remain undetected by a linear method like PPC). Another option is that they might better be explained by early changes in spectral power (see Fig. 2 and the SWD are not sudden and unpredictable phenomena section), rather than by changes in phase relationships as measured with PPC. Moreover it is possible that these pre-ictal changes finally result in the rhythmic cortical and thalamic SWD activity and that only then stable phase-locked rhythmic activity reflected by high PPC values, can be seen.

In all, we propose that the decoupling of the caudal RTN might play a crucial part in the generation of SWD. It prepares the corticothalamo-cortical network for a proepileptic state which starts with decoupling of the cRTN. Pre-ictal decoupling is in good agreement with the descriptions of a pre-ictal long-range desynchronization in humans with absence seizures (Amor et al., 2009).

\section{Frequency and channel specific ictal synchronization}

Changes in ictal PPC were found to be channel-pair and frequency specific. This might indicate differences with respect to the relative contribution of channel-pairs in SWD generation and generalization. In general two noticeable observations could be made:

a) For channel-pairs ctx6-Po, ctx5-Po, and ctx4-Po, synchronization was seen in a huge frequency range $(8-48 \mathrm{~Hz})$, whereas other cortico-thalamic channel-pairs only showed increased PPC values in the SWD specific $8-10 \mathrm{~Hz}$ band and its harmonics. This broad spectral synchronization can be linked to the sharpness of the spikes in both cortex and posterior nucleus. Sharp spikes in the local field potential of SWD are built up by a collection of high frequencies with a similar and constant phase difference. Physiologically, this implies that a huge number of neurons in posterior thalamus and cortex fire in a synchronous burst like fashion. Such a firing pattern is generally believed to be a mechanism of neuronal synchrony and to favor epileptic oscillations (Blumenfeld, 2003). Alternatively, the sharp spikes of the Po nucleus might be the result of a more symmetrical orientation of Po cells, as compared to other thalamic nuclei, which results in strong open field dipoles, easily picked up by the recording electrode. The outcome of studies by Polack et al. (2009), showing Po neurons to be more likely to fire in bursts, phase locked to the spike components of SWD, as compared to VPM cells, and by Cavdar et al. (2012) reporting an increase of so called driver terminals in the posterior nucleus of absence epileptic GAERS rats as compared to healthy Wistar control rats, might be seen to be in line with an important role for the Po in the occurrence of the widespread cortico-thalamo-cortical oscillations named SWD. A differential role of the different thalamic nuclei with respect to the occurrence of SWD was also proposed based on the outcomes of fMRI studies in patients (Tyvaert et al., 2009).

b) Next to this broad synchronization with the deep somatosensory cortex, the Po increased its ictal phase consistency to all other thalamic nuclei. One exception to this was the VPM, which did not show increased PPC values with any other thalamic nuclei and only shows ictal increases in PPC with the cortex. It seems that the VPM stays in a separate loop with the cortical focus whereas the Po takes the role of the major thalamic (resonating) counterpart that takes the responsibility/function of "channeling and synchronization" the thalamic output to the cortex and therefore greatly supports the maintenance of SWDs. This was also proposed in an earlier study by Lüttjohann and van Luijtelaar (2012), who found that the Po was guided by other thalamic nuclei but kept a bidirectional crosstalk with the cortex.

This also implies a relatively minor role of the VPM in SWD occurrence/maintenance as compared to the higher order Po nucleus. It provides/relays the initial, necessary input to the cortical focus, but does not seem to play the primary role in cortico-thalamic resonance feedback. Such a relatively minor role of VPM in SWD occurrence was also indicated by a study of Richards and colleagues (Richards et al., 2003), showing that local injections of Ethosuximide in the VPM/VPL were not very effective in suppressing SWDs; and by studies of Leresche et al. (2012) and Polack et al. (2009) showing that the VPM only rarely fires in a burst-like fashion during SWD. Indeed it is known that the cortico-thalamic neurons from somatosensory cortex layer 6 to VPM are so called 'modulators', which are defined by their action via slow postsynaptic metabotropic receptors and showing weak/ broad input-output firing correlograms, indicating weak responsiveness to cortical input. The Po, by contrast, primarily receives so called driver inputs from somatosensory layer 5 , which are defined by their action via fast acting ionotropic receptors and showing a sharp peak in input-output firing correlograms indicating strong responsiveness to cortical input (Sherman and Guillery, 1998, 2002). Whether local 
application of Ethosuximide to the Po might be more effective to suppress SWD needs to be investigated.

In summary it can be concluded that the Po might be important for the occurrence and generalization of SWD. We propose that this nucleus might be the primary thalamic counterpart to the somatosensorycortex in the generation of cortico-thalamic oscillations of SWD, which is engaged in "channeling and synchronization" of the thalamic output to the cortex and thereby supporting the maintenance of SWD.

\section{SWD are not sudden and unpredictable phenomena}

It is now firmly established that SWD in rodent models are not sudden and unpredictable events. Changes in network communication (see the Network communication during the pre-ictal period: decoupling of the caudal reticular thalamic nucleus section) and spectral (power) SWD precursor activity were found in the current study. Earlier studies reported early, SWD preceding, enhanced firing of neuron in the deep layers of the somatosensory cortex of GAERS rats (Polack et al., 2007), cortical long range desynchronization in humans (Amor et al., 2009) and spectral power changes in humans and animals (Inouye et al., 1992, 1994; Sitnikova and van Luijtelaar, 2009) .

Rather detailed precursor activity was found in LFP recordings of frontal cortex and VPM of WAG/Rij rats, which were uniquely related to the occurrence of SWD (van Luijtelaar et al., 2011a). Time frequency analysis in the current study was performed to validate the existence of such precursor activity (in spectral power) in an extended part of the cortico-thalamo-cortical system with special attention to the deep layers of the somatosensory cortex (the instigator zone of SWD). Looking at this extended network, precursor activity was found to be channel specific. Strong precursor activity was found in the deep layers of the somatosensory cortex. As would be predicted by the cortical focus theory of absence epilepsy, this precursor activity started earlier in the deep somatosensory cortex than in the thalamic recordings. Precursor activity in the cortex and caudal RTN was mostly a combination of delta-theta activity. VPM and Po, on the other hand, predominantly showed precursor activity restricted to the delta band. Whether these channel specific changes in spectral power also affect network communication remains to be investigated by e.g. cross correlation of spectral power.

All in all, the early pre-ictal changes in spectral power (current study, Inouye et al., 1994; Inouye et al., 1992; Sitnikova and van Luijtelaar, 2009; van Luijtelaar et al., 2011b), changes in network communication (current study; Lüttjohann and van Luijtelaar, 2012; Amor et al., 2009; Westmijse et al. (2009); Gupta et al., 2011) and neuronal firing (Polack et al., 2007) raise the possibility of SWD prediction and an early intervention prior to generalization from cortex to thalamus.

\section{A proposed scenario for SWD generation and generalization}

Based on results in the current study and earlier studies in genetic rodent models of absence epilepsy, we propose the following scenario for the generation and generalization of SWD as occurring in WAG/Rij rats:

- The caudal RTN decouples from three structures (VPM, ctx4, Po) that are all part of the somatosensory cortico-thalamo-cortical network. The decoupling prepares the network for a proepileptic state (current study). The decoupling of the cRTN has two consequences which might be prerequisites for SWD generation:

Prerequisite 1 -> Decoupling between cRTN-Po, creates 'idle' Po neurons. In this way the cortical focus can increase its communication with the Po and can quickly entrain it in SWD activity. This probably takes place in a nonlinear fashion (Lüttjohann and van Luijtelaar, 2012).

Prerequisite 2 -> Decoupling of cRTN from VPM and input layer ctx 4 changes the way external stimuli reach the hyperexcitable somatosensory cortex. Input to the deep cortical layers of the somatosensory cortex can then function as an initializing trigger to elicit oscillations in the $7-12 \mathrm{~Hz}$ range, triggering local SWD. This local activity is picked up by the posterior thalamic nucleus and involves the thalamus (see prerequisite 1).

The Po functions as a thalamic reverberator, it keeps a bidirectional crosstalk to the cortex (Lüttjohann and van Luijtelaar, 2012) and may spread SWD activity to remote cortical areas via its widespread connections to the cortex (Kostopoulos, 2001; Polack et al., 2007; Sherman and Guillery, 2002). Furthermore it might be involved in "channeling and synchronization" the thalamic output to the cortex and therefore greatly supporting the maintenance of SWD (current study + Lüttjohann and van Luijtelaar, 2012).

\section{On the validity of extrapolation of findings in animal models to the human condition}

It is crucial to ask how far results on SWD generation from animal models can be extrapolated to the human condition since SWD of rats and humans can easily be seen to differ from each other in terms of their fundamental frequency $(2.5-4 \mathrm{~Hz}$ in humans vs. $7-11 \mathrm{~Hz}$ in rats). Modeling experiments, however, show that this difference can either simply be explained by differences in brain size (with longer axons and dendrites of $\mathrm{C}-\mathrm{T}$ and $\mathrm{T}-\mathrm{C}$ cells in humans as compared to rats and thus longer signal-conduction times) (Roberts and Robinson, 2008), or by a difference in GABA-ergic conductance profiles within the cortico-thalamo-cortical system (in species with a $\mathrm{GABA}_{\mathrm{B}}$ conduction dominance showing 2.5-4 Hz SWD and species with a $\mathrm{GABA}_{\mathrm{A}}$ conduction dominance showing 6-10 Hz SWD) (Destexhe, 1999).

In general the genetic rat models like the WAG/Rij rat, used in this study, and GAERS rats, are the best characterized models for absence epilepsy in humans and are shown to possess a high face and predictive validity as reviewed in detail in (Marescaux et al., 1992; Coenen and van Luijtelaar, 2003; van Luijtelaar and Sitnikova, 2006; van Luijtelaar et al., 2011b).

In terms of brain networks, it can be noted that for children with childhood absence epilepsy also, more and more evidence accumulates that SWD are not a 'primary generalized' type of seizure but that there are local, cortical, susceptible regions that functions as the initiator of SWD and then rapidly entrains selective corticothalamo-cortical networks to form a resonator circuitry for SWD generalization and maintenance (Gupta et al., 2011; Tyvaert et al., 2009; Westmijse et al., 2009). It needs to be pointed out, however, that the location of this susceptible zone in children was found to be located in the parietal and frontal cortex and not in the somatosensory cortex as is the case for the genetic rat models. Consequently, the exact secondarily involved thalamic nuclei might also be different.

The basic principles, however, as outlined in detail for the WAG/Rij rat in the A proposed scenario for SWD generation and generalization section, are likely to be the same or at least similar. A pre-SWD change in the inhibitory and excitatory balance in the connected brain network (decoupling of the RTN), enables a situation of increased communication between a susceptible zone and its anatomically connected thalamic counterparts (prerequisite 1 ). It enables that also a trigger-input can reach this susceptible zone (prerequisite 2) starting a localized epileptic discharge that is picked up and generalized by the thalamic counterpart. Whether in the human condition this thalamic counterpart is also a higher order thalamic nucleus (as the Po found for the WAG/Rij rat) needs to be investigated. Interestingly, a recent imaging study (Tyvaert et al., 2009) inspecting activation patterns of different thalamic nuclei in children with childhood absence epilepsy reported early increases of the fMRI bold signal for the centromedian-parafascicular nuclei (two higher order nuclei) as compared to a later activation of the anterior nucleus.

Likewise, the RTN is reported to monitor and affect all reciprocal communications between thalamus and cortex and plays a crucial 
role in all theoretical models (an exception is the bicuculline model in cats, Steriade) on how absences are generated (Guillery et al., 1998; Huguenard and McCormick, 2007; Meeren et al., 2005). However, our data suggest a more complex and even an opposite role for the caudal part of the RTN as was previously assumed. Certainly, however, future translational studies, preferably with improved non-invasive imaging and source localization methods, are desirable to establish network mechanisms in patients.

In summary: via combined investigation of dynamics of spectral power in individual channels and network communication (synchrony/stability of phase relationships) between channels in the corticothalamo-cortical system of absence epileptic WAG/Rij rats as seen during SWD generation, the following conclusions can be drawn:

- SWD do not occur sudden but are preceded by changes in network communication and spectral (power) precursor activity of pre-ictal LFPs.

- Spectral precursors are clearly present in the instigator zone of SWD, which is in good agreement with the cortical focus theory of absence epilepsy.

- A pre-ictal decoupling of the cRTN with other thalamic nuclei and cortex might be a prerequisite for SWD generation, which prepares the cortico-thalamic-cortical network in a pro-epileptic state.

- The posterior thalamic nucleus shows strongest (all frequencies) and widest (to all other channels) increases in network synchrony and might therefore be regarded as a key player for SWD generation and maintenance.

\section{Acknowledgments}

The authors gratefully acknowledge the support of Gerard van Oijen (technical assistance); Norbert Hermesdorf (construction of electrode system units); Hans Krijnen and Saskia Menting-Hermeling (biotechnical assistance and animal care).

\section{Grants}

The study was partially financed by the Donders Centre for Cognition and the BrainGain Smart Mix Program of the Netherlands Ministry of Economic Affairs and the Netherlands Ministry of Education, Culture and Science.

\section{Disclosure}

None of the authors has any conflict of interest to disclose. All authors have approved the final article.

\section{References}

Abbasova, K.R., et al., 2010. The role of perioral afferentation in the occurrence of spikewave discharges in the WAG/Rij model of absence epilepsy. Brain Res. 1366, 257-262.

Aker, R.G., et al., 2006. GABA(A) receptor mediated transmission in the thalamic reticular nucleus of rats with genetic absence epilepsy shows regional differences: functional implications. Brain Res. 1111, 213-221.

Amor, F., et al., 2009. Cortical local and long-range synchronization interplay in human absence seizure initiation. NeuroImage 45, 950-962.

Avoli, M., et al., 1983. An analysis of penicillin-induced generalized spike and wave discharges using simultaneous recordings of cortical and thalamic single neurons. J. Neurophysiol. 50, 819-837.

Berg, A.T., et al., 2010. Revised terminology and concepts for organization of seizures and epilepsies: report of the ILAE Commission on Classification and Terminology, 2005-2009. Epilepsia 51, 676-685.

Blumenfeld, H., 2003. From molecules to networks: cortical/subcortical interactions in the pathophysiology of idiopathic generalized epilepsy. Epilepsia 44 (Suppl. 2), 7-15.

Bosnyakova, D., et al., 2006. Time-frequency analysis of spike-wave discharges using a modified wavelet transform. J. Neurosci. Methods 154, 80-88.

Buzsaki, G., 2006. Rhythms of the Brain. Oxford University Press.
Capovilla, G., et al., 2009. Conceptual dichotomies in classifying epilepsies: partial versus generalized and idiopathic versus symptomatic (April 18-20, 2008, Monreale, Italy). Epilepsia 50, 1645-1649.

Cavdar, S., et al., 2012. Do the quantitative relationships of synaptic junctions and terminals in the thalamus of genetic absence epilepsy rats from Strasbourg (GAERS) differ from those in normal control Wistar rats. Neurol. Sci. 33, 251-259.

Coenen, A.M., 1995. Neuronal activities underlying the electroencephalogram and evoked potentials of sleeping and waking: implications for information processing. Neurosci. Biobehav. Rev. 19, 447-463.

Coenen, A.M., van Luijtelaar, E.L., 2003. Genetic animal models for absence epilepsy: a review of the WAG/Rij strain of rats. Behav. Genet. 33, 635-655.

Crick, F., 1984. Function of the thalamic reticular complex: the searchlight hypothesis Proc. Natl. Acad. Sci. U. S. A. 81, 4586-4590.

Depaulis, A., van Luijtelaar, G., 2006. Genetic models of absence epilepsy. In: Pitkanen, A., Schwartzkroin, P.A., Moshe, S.L. (Eds.), Models of Seizures and Epilepsy. Elsevier Academic Press, San Diego.

Destexhe, A., 1999. Can GABAA conductances explain the fast oscillation frequency of absence seizures in rodents? Eur. J. Neurosci. 11, 2175-2181.

Drinkenburg, W.H., et al., 1991. Spike-wave discharges and sleep-wake states in rats with absence epilepsy. Epilepsy Res. 9, 218-224.

Guillery, R.W., et al., 1998. Paying attention to the thalamic reticular nucleus. Trends Neurosci. 21, 28-32.

Gupta, D., et al., 2011. Space-time network connectivity and cortical activations preceding spike wave discharges in human absence epilepsy: a MEG study. Med. Biol. Eng. Comput. 49, 555-565.

Huguenard, J.R., McCormick, D.A., 2007. Thalamic synchrony and dynamic regulation of global forebrain oscillations. Trends Neurosci. 30, 350-356.

ILAE, 1989. Proposal for revised classification of epilepsies and epileptic syndromes Commission on Classification and Terminology of the International League Against Epilepsy. Epilepsia 30, 389-399.

Inouye, T., et al., 1992. Abnormality of background EEG determined by the entropy of power spectra in epileptic patients. Electroencephalogr. Clin. Neurophysiol. 82, 203-207.

Inouye, T., et al., 1994. Increases in the power spectral slope of background electroencephalogram just prior to asymmetric spike and wave complexes in epileptic patients. Neurosci. Lett. 173, 197-200.

Kostopoulos, G.K., 2001. Involvement of the thalamocortical system in epileptic loss of consciousness. Epilepsia 42 (Suppl. 3), 13-19.

Le Van Quyen, M., 2005. Anticipating epileptic seizures: from mathematics to clinical applications. C. R. Biol. 328, 187-198.

Leresche, N., et al., 2012. From sleep spindles of natural sleep to spike and wave discharges of typical absence seizures: is the hypothesis still valid? Pflugers Arch. 463, 201-212.

Lüttjohann, A., van Luijtelaar, G., 2012. The dynamics of cortico-thalamo-cortical interactions at the transition from pre-ictal to ictal LFPs in absence epilepsy. Neurobiol Dis. 47, 49-60.

Lüttjohann, A., et al., 2011. Electrical stimulation of the epileptic focus in absence epileptic WAG/Rij rats: assessment of local and network excitability. Neuroscience $188,125-134$

Marescaux, C., et al., 1992. Genetic absence epilepsy in rats from Strasbourg - a review. J. Neural Transm. Suppl. 35, 37-69.

Maris, E., Oostenveld, R., 2007. Nonparametric statistical testing of EEG- and MEG-data J. Neurosci. Methods 164, 177-190.

Maris, E., et al., 2007. Nonparametric statistical testing of coherence differences. J. Neurosci. Methods 163, 161-175.

Meeren, H.K., et al., 2002. Cortical focus drives widespread corticothalamic networks during spontaneous absence seizures in rats. J. Neurosci. 22, 1480-1495.

Meeren, H., et al., 2005. Evolving concepts on the pathophysiology of absence seizures: the cortical focus theory. Arch. Neurol. 62, 371-376.

Meeren, H.K., et al., 2009. Thalamic lesions in a genetic rat model of absence epilepsy: dissociation between spike-wave discharges and sleep spindles. Exp. Neurol. 217, 25-37.

Mormann, F., et al., 2003a. Epileptic seizures are preceded by a decrease in synchronization. Epilepsy Res. 53, 173-185.

Mormann, F., et al., 2003b. Automated detection of a preseizure state based on a decrease in synchronization in intracranial electroencephalogram recordings from epilepsy patients. Phys. Rev. E Stat. Nonlin. Soft Matter Phys. 67, 021912.

Oostenveld, R., et al., 2011. FieldTrip: open source software for advanced analysis of MEG EEG, and invasive electrophysiological data. Comput. Intell. Neurosci. 2011, 156869.

Paxinos, G., Watson, C., 1998. The Rat Brain in Stereotaxic Coordinates. Academic Press, SanDiego.

Pinault, D., 2004. The thalamic reticular nucleus: structure, function and concept. Brain Res. Brain Res. Rev. 46, 1-31.

Pinault, D., O'Brien, T.J., 2005. Cellular and network mechanisms of geneticallydetermined absence seizures. Thalamus Relat. Syst. 3, 181-203.

Pinault, D., et al., 2001. Medium-voltage 5-9-Hz oscillations give rise to spike-and-wave discharges in a genetic model of absence epilepsy: in vivo dual extracellular recording of thalamic relay and reticular neurons. Neuroscience 105, 181-201.

Pinault, D., et al., 2006. Corticothalamic 5-9 Hz oscillations are more pro-epileptogenic than sleep spindles in rats. J. Physiol. 574, 209-227.

Polack, P.O., et al., 2007. Deep layer somatosensory cortical neurons initiate spikeand-wave discharges in a genetic model of absence seizures. J. Neurosci. 27, 6590-6599.

Polack, P.O., et al., 2009. Inactivation of the somatosensory cortex prevents paroxysmal oscillations in cortical and related thalamic neurons in a genetic model of absence epilepsy. Cereb. Cortex 19, 2078-2091. 
Proulx, E., et al., 2006. Functional contribution of specific brain areas to absence seizures: role of thalamic gap-junctional coupling. Eur. J. Neurosci. 23, 489-496.

Richards, D.A., et al., 2003. Targeting thalamic nuclei is not sufficient for the full anti-absence action of ethosuximide in a rat model of absence epilepsy. Epilepsy Res. 54, 97-107.

Roberts, J.A., Robinson, P.A., 2008. Modeling absence seizure dynamics: implications for basic mechanisms and measurement of thalamocortical and corticothalamic latencies. J. Theor. Biol. 253, 189-201.

Schnitzler, A., Gross, J., 2005. Normal and pathological oscillatory communication in the brain. Nat. Rev. Neurosci. 6, 285-296.

Seidenbecher, T., et al., 1998. Relations between cortical and thalamic cellular activities during absence seizures in rats. Eur. J. Neurosci. 10, 1103-1112.

Sherman, S.M., Guillery, R.W., 1998. On the actions that one nerve cell can have on another: distinguishing "drivers" from "modulators". Proc. Natl. Acad. Sci. U. S. A. 95, 7121-7126.

Sherman, S.M., Guillery, R.W., 2002. The role of the thalamus in the flow of information to the cortex. Philos. Trans. R. Soc. Lond. B Biol. Sci. 357, 1695-1708.

Sitnikova, E., van Luijtelaar, G., 2006. Cortical and thalamic coherence during spikewave seizures in WAG/Rij rats. Epilepsy Res. 71, 159-180.

Sitnikova, E., van Luijtelaar, G., 2009. Electroencephalographic precursors of spikewave discharges in a genetic rat model of absence epilepsy: Power spectrum and coherence EEG analyses. Epilepsy Res. 84, 159-171.
Smyk, M.K., et al., 2011. Endogenous rhythm of absence epilepsy: relationship with general motor activity and sleep-wake states. Epilepsy Res. 93, 120-127.

Sohal, V.S., et al., 2003. Dynamic GABA(A) receptor subtype-specific modulation of the synchrony and duration of thalamic oscillations. J. Neurosci. 23, 3649-3657.

Tyvaert, L., et al., 2009. Thalamic nuclei activity in idiopathic generalized epilepsy: an EEG-fMRI study. Neurology 73, 2018-2022.

van Luijtelaar, G., Sitnikova, E., 2006. Global and focal aspects of absence epilepsy: the contribution of genetic models. Neurosci. Biobehav. Rev. 30, 983-1003.

van Luijtelaar, G., et al., 2011a. Spike-wave discharges in WAG/Rij rats are preceded by delta and theta precursor activity in cortex and thalamus. Clin. Neurophysiol. 122, 687-695.

van Luijtelaar, G., et al., 2011b. On the origin and suddenness of absences in genetic absence models. Clin. EEG Neurosci. 42, 83-97.

Vergnes, M., et al., 1989. Interhemispheric desynchronization of spontaneous spike-wave discharges by corpus callosum transection in rats with petit mal-like epilepsy. Epilepsy Res. 4, 8-13.

Vinck, M., et al., 2010. The pairwise phase consistency: a bias-free measure of rhythmic neuronal synchronization. NeuroImage 51, 112-122.

Westmijse, I., et al., 2009. Onset and propagation of spike and slow wave discharges in human absence epilepsy: a MEG study. Epilepsia 50, 2538-2548. 